\title{
Comparative study between transmission line theory and the electromagnetic field approach in grounding system analysis
}

\author{
Mabrouki. $\mathrm{H}^{1}$, Derbel. $\mathrm{N}^{2}$, Hadj Abdallah $\mathrm{H}^{3}$ \\ Advanced Command and Energy Management Laboratory, Engineering National School of Sfax. \\ Sfax University, Tunisia.
}

\begin{abstract}
This paper present a study of the grounding system transient behavior. A comparative study between the transmission line theory and the electromagnetic field approach is taken account, with an aim to know the advantages and disadvantages of each method; in order to have an accurate high frequency analysis for grounding system.
\end{abstract}

KEYWORDS: Grounding systems analysis, transmission line model, and electromagnetic field approach.

\section{Introduction}

In the literature we find a lot of paper describing the performance of wire conductors energized at low and high frequencies in space, air and soil.

Generally, those work has concentrated on the response metallic structures exited by a voltage applied to two closely spaced (but isolated) feed point terminals locate on the energized structures or by an incident electromagnetic field illuminating the structures [1]

Many authors are tried seriously to find a solution for to transient problems for a grounding systems.

The most famous work involving high frequency performance of system grounding structures was conducted by SUNDE [2].

In order to analyze the ground systems, mathematical models are used to more accurately determine the electric field and current distribution in and around these systems. The importance of this study comes from the role of grounding systems in the protection of electrical installations and power station.

We can also study the frequency behavior of those systems, especially in case of an electric default (lightning), that means a high frequencies analysis.

In this context, we find several publications that reflect the analysis of grounding systems at high frequencies using various methods.

These include for example the work of Miliopoulos and Valasquez who proposed a solution in time domain based on line theory, this approach is valid for long horizontal conductors but not for vertical and arbitrary related conductors [3].

Another analysis offered by Takashima [4], shows the effect of the permittivity of the ground, without taking into account the important effect of induction [3].

For his part, Papalexoupoulos [5] used an approach in the time domain but for low frequencies in order to neglect the electric field component at integration [3].

One of those important works is presented by Dawalibi $[6,7]$, whose give an analysis of the response of practical grounding grid, using electromagnetic field theory in the frequency domain, a large frequency interval was taken account. Furthermore, to avoid direct numerical integration of the general solution, the analytical expressions used in computer model were derived based on the quasi-static assumption [3].

After that, Leonid Grcev make a logical analyze for this problem, so he developed in his $\mathrm{PhD}$ thesis [8] the methodology for the analysis of an arbitrary grounding conductor at all frequencies based on computational techniques involving the method of moment, numerical integration of Sommerfeld integrals and Fast Fourier Transform (FFT) .To exploit this work, Grcev was studied a horizontal linear conductor [9].

This paper gives a simple comparison between the electromagnetic field approach and the transmission line theory by exploiting studies gives in works presented previously; and in order to simplify the transient analysis of the grounding system.

\section{The electromagnetic field approach}

After a general study, we can say that the electromagnetic field approach is the most accurate method for modeling the transient behavior of grounding system.

This importance exists when this approach solves full Maxwell's equation with minimum approximations.

There in two principal methods in order to implement this approach, the first one is the Method of Moment (MoM), the second one is the Finite Element Method (FEM).

Grcev is the first one who's developed the model for transient behavior of a grounding system. This method starts from electric field Maxwell's integral equation as given in (1) [10]:

$$
\begin{aligned}
& E^{s}=\frac{1}{4 \pi j \omega \dot{\varepsilon}}\left(\nabla \nabla-\gamma^{2}\right) \int_{l} t^{\prime} I_{1}\left(r^{\prime}\right) G_{n}\left(r, r^{\prime}\right) d l \\
& G_{n}\left(r, r^{\prime}\right)=G_{l}\left(r, r^{\prime}\right)+G_{i}\left(r, r^{\prime}\right)+G_{s}\left(r, r^{\prime}\right)
\end{aligned}
$$

With;

$E^{s}:$ is the total scattered electric field along the surface of the conductor.

$$
\begin{aligned}
& t^{\prime} I_{1}\left(r^{\prime}\right): \text { is the current flowing among the conductor. } \\
& \dot{\varepsilon}=\varepsilon+\frac{\sigma}{j \omega}: \text { is the complex medium permittivity. }
\end{aligned}
$$


$\gamma^{2}=j \omega \mu(\sigma+j \omega \varepsilon)$; is the wave propagation constant in the medium.

$\sigma, \quad \varepsilon$ and $\mu$ as conductivity permittivity and permeability, respectively.

$G_{n}\left(r, r^{\prime}\right):$ is the complete Green function.

$G_{l}\left(r, r^{\prime}\right)$ And $G_{i}\left(r, r^{\prime}\right)$ are Green's dyadic functions for the electric field at $r$ due to the current element and its image [11].

$G_{s}\left(r, r^{\prime}\right)$ is a correction term (expressed in terms of Sommerfeld integrals).

In formulating (2) the calculation of the Sommerfeld integral is complex; most models used a simplification in order to take on account the interfaces between different medium.

The boundary condition is that the total longitudinal electric field on the surface of the grounding conductor should satisfy equation (3).

$t\left(E^{i}+E^{s}\right)=I Z_{s} \Delta L$

With:

$E^{i}:$ is the incident electric field.

$Z_{s}$ : is the per-unit length series impedance of the conductor including skin effect.

The Method of Moment (MoM) is the appropriate method for the numerical treatment of equation (3).

MoM is based on transforming the associated integral equation to a system of linear algebraic equations with $\mathrm{N}$ unknowns. (N represents the coefficients of the current base on some appropriate expansions).

For a grounding system, the distribution of the current along the conductor is primordial and is used in the system analysis.

However, this distribution is usually hard to be exactly modeled, so that it is necessary to use some assumptions.

Among those approximations we can distinguish two principal which help us to find solution for the integral field equation (1):

The first one is called the Reaction Integral Equation (RIE), this kind of integration assume that the distribution of the current among the conductor as piecewise sinusoidal.

The second one is the mixed potential equation (MPIE) it is used when the current distribution is approximated as a piecewise constant.

In literature [12] we find an example of linear algebraic expression of equation (2) based on MPIE is given by equation (4);

$$
\left[\begin{array}{cccc}
1 & 0 & \ldots & 0 \\
Z_{21} & Z_{22}+Z_{s} \Delta L & \ldots & Z_{2 N} \\
\vdots & \vdots & \vdots & \vdots \\
Z_{N 1} & Z_{N 2}+Z_{s} \Delta L & \ldots & Z_{N N}+Z_{s} \Delta L
\end{array}\right] \cdot\left[\begin{array}{l}
I_{1} \\
I_{2} \\
\vdots \\
I_{N}
\end{array}\right]=\left[\begin{array}{l}
I_{g} \\
0 \\
\vdots \\
0
\end{array}\right]
$$

With $Z_{n m}$ refers to mutual impedance, which is equal to the voltage across the m.th segment due to a unit current through the n-th segment.

And $I_{g}$ is the first segment injection current. $Z_{n m}$ can be calculated by the following equation (5):

$$
Z_{n m}=\int_{l_{n}} F_{n}^{d}\left(l_{n}\right) \vec{l}_{n} \cdot \vec{E}_{m} d l
$$

In fact, the current expansion function for the segment $n$ is designed by $F_{n}^{d}\left(l_{n}\right)$ and the average electric field on surface of the segment $n$ due to the current in segment $m$.

The mutual impedance $Z_{n m}$ is dependent only on the geometry of the system, the frequency and the electric characteristics of soil (the resistivity $\rho$, the permittivity $\varepsilon$ and the permeability $\mu$ ).

In order, to get the current distribution along the grounding conductor, we have to solve the equation (4) in frequency domain.

By using the fundamental equations for the related source and medium, and if the currents sources for every segments of grounding conductors are known, the electric field around the grounding system and the leakage current from the grounding conductor segment to the soil can be simply calculated.

At deferent points on the surface of the grounding conductor, the potential could be exactly calculated by integration of the normal electric field from the point on the surface of the conductor to the remote earth.

In this analysis, the longitudinal component of the electric field is not incorporated because its contribution is insignificant compared to that of normal electric field especially for lightning studies (high frequency domain). Finally, to get response in time domain, we could use a suitable Fourier inversion technique. The response to a steady-state, time harmonic excitation is computing for a wide range of frequency [3]. In fact, a transfer function can be constructed for every frequency considered. The geometric and electromagnetic properties of the grounding system and its environment; are two important parameters which differentiate the transfer function. Since, with minimum approximations, electromagnetic field approach based on Method of Moment solves full Maxwell's equations in frequency domain.

Consequently it is believed to be accurate, despite the high input frequency of sources, the electromagnetic field approach is the most accurate. However, this model is too complex to be implemented.

Further when we have a grounding system with a large structure; the computation time is very large.

Besides, the model issue of the electromagnetic field approach cannot be easily modified to include nonlinearity due to soil ionization, and combine other nonlinear devices that have time domain models.

Among many works, we find those of Nekhoul [13] [14] who have developed a model basing on the electromagnetic field approach. This model starts from 
electric or magnetic energy equation which involves partial deferential Maxwell's equations with respect to the vector potential $(\vec{A})$ and the scalar potential $(V)$ in different domains/volumes of the system. So we find the following equations:

$$
\begin{aligned}
& \int_{\Omega}\left(\frac{1}{\mu_{0}}(\nabla \times \vec{W}) \cdot(\nabla \times \vec{A})+\frac{1}{\mu_{0}}(\nabla \times \vec{W}) \cdot(\nabla \times \vec{A})+\right. \\
& \left.\left(\sigma_{\text {soil }}+j \omega \varepsilon_{\text {soil }}\right)(j \omega \vec{W} \cdot \vec{A}+\vec{W} \cdot \nabla V)\right) d \Omega=0 \\
& \int_{\Omega}\left(\sigma_{\text {soil }}+j \omega \varepsilon_{\text {soil }}\right) \cdot \nabla \varepsilon \cdot(j \omega \cdot \vec{A}+\nabla V) d \Omega \\
& \int_{\Omega}\left(\frac{1}{\mu_{0}}(\nabla \times \omega) \cdot(\nabla \times \vec{A})+\frac{1}{\mu_{0}}(\nabla \times \vec{W}) \cdot(\nabla \times \vec{A})\right) d \Omega=0
\end{aligned}
$$

With:

$\vec{W}$ and $\omega$ are the weighting functions for the vector potential and scalar potential, respectively.

Those equations were transformed to linear equation by dividing the whole system into $N$ small volumes or elements; in order to solve the problem numerically. So that, by using electromagnetic field approach based on FEM (Finite Element Method) we obtain the discretization

of the domain (geometry or medium) problem can be highly flexible non-uniform patches or elements.

This advantage can be taken account in order to include the soil ionization into the above said model; this method present an inconvenient which is the use of Method of Moment to solves indirectly the complex Maxwell's equations.

\section{Transmission line approach:}

This approach considers the grounding system as a line, or many lines. Sunde is the first to have proposed in [15] to model a buried conductor by a line.

Initially, this approach was used to model the Transient behavior of a buried counterpoise. The grounding system models derived from the line theory are more stringent than those derived from circuit theory; especially at high frequencies because they take into account the propagation phenomena. Furthermore, for simple earthed system, they are easily exploitable by software for calculating transient networks, which are able to model lines. Finally, some of these models take into account nonlinear phenomena in the soil (soil ionization). However, this approach is not the best for a complex structure, for which the number of lines becomes important and the parameters $\mathrm{R}$ ', L', C 'and G' becomes difficult to assess without using a finite element modeling. So we say that the electromagnetic models are the most rigorous theoretical level.

On the other hand, by using the transmission line theory we can create a model which predicts all the important features of transient behavior of grounding systems. Unluckily, the development of this approach was not as fast as electromagnetic approach.

In fact, all the transmission lines approaches were limited to model simple grounding systems, i.e. counterpoise wires or single grounding rods.

In fallowing, we take the example of a simple grounding system which is an earthling electrode. So, in order to be near the reality, we consider that the electrode has three components:

\section{$\checkmark$ Resistive \\ $\checkmark$ Capacitive \\ $\checkmark$ Inductive}

Many works choose the Transmission Line Theory to develop a circuit model (lumped R L C, pi section) [16]; although there are many disadvantages of conventional transmission line approach with uniform per-unit length parameters which more developed in [17].

Besides, there is the non-uniform transmission line approach for modeling the transient behavior of grounding system, this kind of approach is largely detailed on [18].

\section{Conclusion}

After this simple bibliographic study, we can conclude that, firstly the electromagnetic approach, because of its complicated mathematical expressions, is difficult to understand. And it has complicated solution procedure and a difficulty to include the soil ionization. Furthermore this approach require very powerful computer because of the large computation time. The most advantage is that this approach is the most accurate approach.

Secondly, the transmission line approach is considered like the simplest in point of view of mathematical expression and also it is very easy to appreciate. Besides, this approach has a very simple solution procedure which also can easily include the soil ionization and can predict the wave propagation delay. And because of the small computation time of Transmission line approach we can use normal PC. Relative to the electromagnetic approach, the TL approach is less accurate with a reasonably accuracy.

\section{REFERENCES}

[1] A. T. Adams, B. J. Strait, D. E. Warren, D. C. Kuo, T.E. Baldwin, «Near fields of Wire Antennas by Matrix Methods», IEEE Transactions on Antennas and Propagation, Vol. AP-21, 602-610, September 1973.

[2] E. D. Sunde, " Earth Conduction Eflects in Transmission Systems», D. Van Nostrand Company, Inc., New York 1949.

[3] L.Grcev, F Dawalibi « An electromagnetic model for transients in grounding systems» IEEE Trans. Power Delivery, 5 (1990), pp. 1773-1781.

[4] T. Takashima, T. Nakae, R. Ishibashi, « High Frequency Characteristics of Impedances to Ground and Field Distributions of Ground Electrodes», IEEE Transactions on Power 
Apparatus and Systems, Vol. PAS-100, No. 4, April 1981, pp1893-1900.

[5] A. D. Papalexopoulos, A. P. Meliopoulos, «Frequency Dependent Characteristics of Grounding Systems», IEEE Transactions on Power Delivery, Vol PWRD-2, 1073-1081. October1987.

[6] F. Dawalibi, «Electromagnetic Fields Generated by Overhead and Buried Conductors. Part 1 - Single Conductor. Part $2 \sim$ Ground Networks», IEEE Transactions on Power Delivery, Vol PWRD-1, 105-119, October 1986.

[7] F. Dawalibi, « Effectiveness of Station Grounding and Surface Detection of Damaged Ground Conductors», Canadian Electrical Association, Report 019T218, April 1984.

[8] L. Grcev, «Calculation of the Transient Impedance of Grounding Systems», D. Sc., thesis (in SerboCroatian), University of Zagreb, Yugoslavia 1986.

[9] L. Grcev, Z. Haznadar, «A Novel Technique of Numerical Modeling of Impulse Current Distribution in Grounding Systems, Proceedings»,19th International conference on lightning protection, Graz 1988, paper 3.4, 165169.

[10] L. Grcev, M. Heimbach, "Frequency Dependent and Transient Characteristics of Substation Grounding Systems," IEEE Transaction on Power Delivery, Vol. 12, pp. 172-178, No. 1, January 1997 (also presented at IEEE/PES 1996 Summer Meeting, Denver, USA,July.

[11] G.J. Burke, E. K. Miller, «Modeling Antenna near to and Penetrating a Lossy Interface», IEEE Transactions on Antennas and Propagation, vol. AP32, pp 1040-1049, oct.1984.
[12] J. H. Richmond, « Radiation and scattering by thin wire structure in a homogeneous conducting medium » IEEE Trans. Antenna and Propagation, Vol .22, pp 365 , March 1974.

[13] B. Nekhoul, C.Curein, P.Labie, G.Meunier and R. Feuillet, «A finite element method for calculating fields generated substation grounding systems» IEEE Transaction .on magnetic, vol 31 , No 3 pp 2150-2155,May , 1995 .

[14] B. Nekhoul, P.Labie, and G.Meunier « Calculating the impedance of grounding system» IEEE Transaction .on magnetic, vol 32 , No 3 pp 150915123 ,May , 1996.

[15] E. D. Sunde, "Earth conduction effects in transmission systems", Dover Edition, 1967.

[16] Y Liu , M. Zitnik and R. Thottapillil « An improved transmission line model of grounding system» IEEE Transaction on EMC, vol43, No. 3, page.348-355, Aug 2001.

[17] Y Liu, M. Zitnik and R. Thottapillil « A time domain transmission line model of grounding system» $12^{\text {th }}$ international Symposium on High Voltage Engineering, Bangalore, India August 2428,2001, p.154-157.

[18] Y Liu, N. Theethayi and R. Thottapillil« Nonuniform transmission line approach for transient analysis of grounding system under lightning impulse $\gg 17^{\text {th }}$ international Conference on lightning protection, Avignon, France $13^{\text {th }}-16^{\text {th }}$ September , 2004 paper No 147. 